\title{
VEGF-Induced Cytoskeletal Alterations Relating to Vascular Permeability in Endothelial Cells
}

\author{
Kathryn S.R. Spencer and David A. Cheresh \\ Department of Immunology, The Scripps Research Institute, La Jolla, CA 92037
}

Endothelial cells within blood vessels constitute a barrier between circulating plasma proteins and surrounding tissues. The integrity of this barrier maintains the homeostasis of fluid and ions in the perivascular space. If this barrier is disrupted, vascular permeability increases, and severe edema can occur (1). Increased vascular permeability has been associated with stroke (2), myocardial infarction (3), and solidtumor cancers (4). For example, in stroke, neuronal damage occurs in part because of increased edema from permeable blood vessels (5).

Vascular endothelial growth factor (VEGF), also known as vascular permeability factor (VPF) (6), is produced in response to ischemia associated with stroke (7) and myocardial infarction (8). This results in increased vascular permeability to small proteins and ions, resulting in edema. VEGF is known to stimulate the Src-family kinase pathway within endothelial cells (9). In fact, mice deficient in Src show a selective loss of VEGF-mediated permeability. This was confirmed using a Src inhibitor which, when administered to genetically normal mice, suppressed VEGFmediated vascular permeability, resulting in decreased edema flowing stroke (10).

Our interest lies in further defining the molecular basis of VEGF-mediated Srcdependent vascular permeability. Presumably, VEGF-mediated Src activation leads to phosphorylation of proteins directly or indirectly capable of regulating cell-cell adhesions, including the cadherin-associated adherens junctions. Adherens junctions are actin-associated multi-protein complexes found at the cell surface forming cell-cell junctions. Therefore, examination of actin cytoskeletal dynamics is of interest in the context of VEGF-regulated adherens junction breakdown.

We have undertaken studies involving live-cell time-lapse confocal analysis of endothelial actin cytoskeletal dynamics at cell-cell junctions before and after VEGF treatment. Endothelial cells electroporated with a GFP-actin fusion construct were treated with VEGF, and we monitored cell-cell junctions and actin fiber assembly. Initially, endothelial cells show limited stress fibers and ruffle profusely, demonstrating non-static cell-cell adhesions. Immediately after VEGF application, cells decrease ruffling, and form more stress fibers. Blocking Src activity with specific pharmacological agents altered the VEGF-mediated actin assembly pattern and the junctions associated with these cells. These findings suggest that VEGF-mediated Src activation may influence endothelial cell-cell junctional activity and vascular permeability by altering the actin cytoskeleton. These live-cell studies complement ongoing biochemical analyses of VEGF signaling pathways in vivo and in vitro. 
1. L. Schilling, M. Wahl, Adv Exp Med Biol 474, 123-41 (1999).

2. G. A. Rosenberg, Prog Cardiovasc Dis 42, 209-16 (Nov-Dec, 1999).

3. J. C. Nilsson et al., Heart 85, 639-42 (Jun, 2001).

4. R. F. Del Maestro et al, Can J Neurol Sci 17, 177-83 (May, 1990).

5. Z. G. Zhang et al., J Clin Invest 106, 829-38 (Oct, 2000).

6. D. R. Senger et al., Science 219, 983-5 (Feb 25, 1983).

7. M. Slevin et al., Stroke 31, 1863-70 (Aug, 2000).

8. X. Xu et al, J Thorac Cardiovasc Surg 121, 735-42 (Apr, 2001).

9. B. P. Eliceiri et al., Mol Cell 4, 915-24 (Dec, 1999).

10. R. Paul et al., Nat Med 7, 222-7 (Feb, 2001).
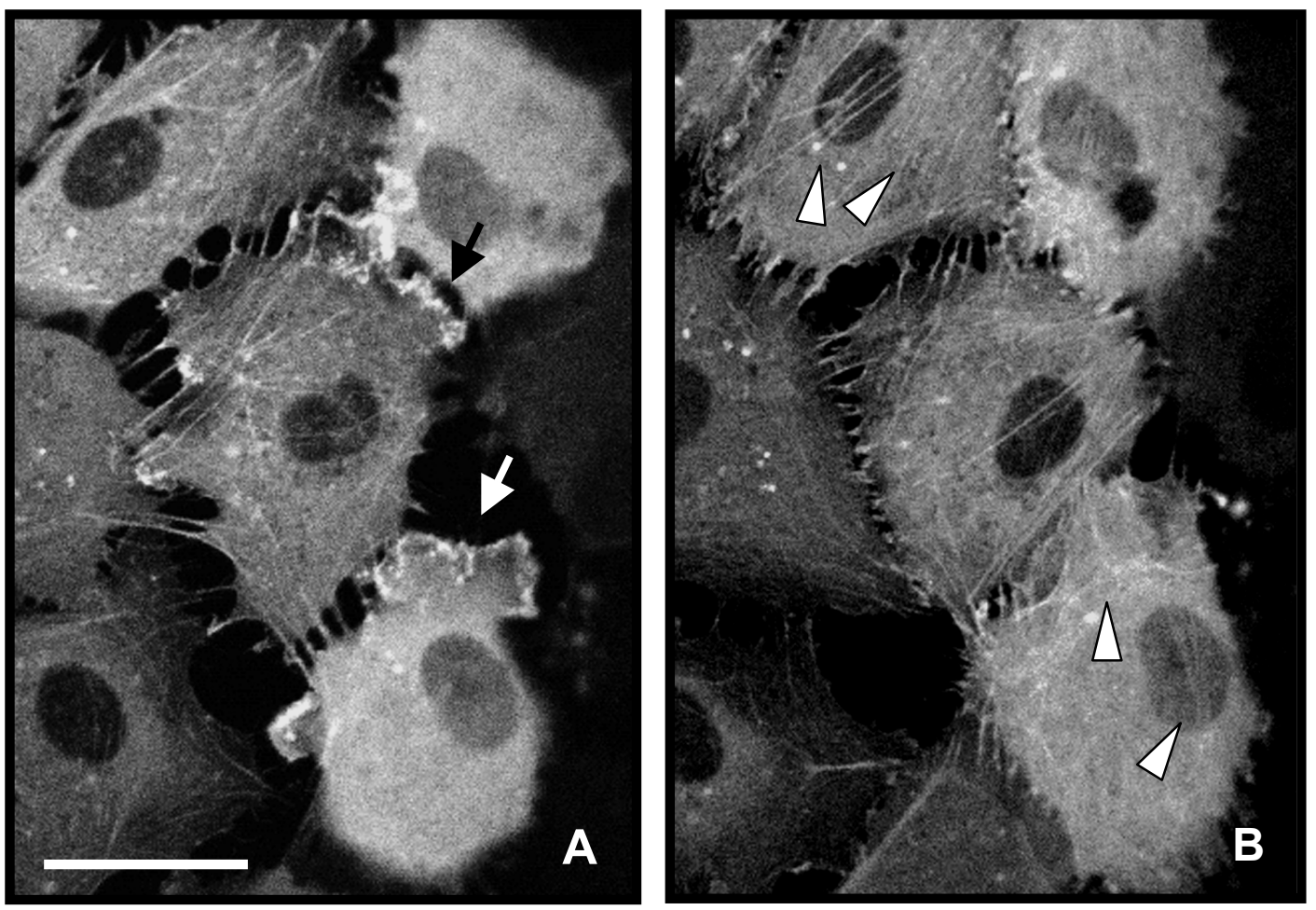

Figure 1. Endothelial cells expressing GFP-actin before (A) and after (B) treatment with 50ng/ml VEGF. Notice ruffling in non-treated cells (arrows), and an increase in actin stress fibers (arrowheads) in VEGF-treated cells. Bar $=50 \mu \mathrm{m}$ 